\title{
Transcultural Adaptation of DCDQ In Kerala, India
}

\author{
(Dec 2020) \\ Prof Mrs Elizabeth Varkey, Prof (Dr) Roy K George \\ Baby Memorial College of Nursing, Kozhikode,Kerala \\ DOI: 10.29322/IJSRP.11.12.2021.p12008 \\ http://dx.doi.org/10.29322/IJSRP.11.12.2021.p12008
}

\begin{abstract}
Developmental Coordination Disorder (DCD) has gained attention worldwide, but little or less awareness is elicited in Kerala regarding the same. The revised DCD Questionnare is one of the most commonly utilized screening tools for identification of DCD in children. The aim of this study was to translate the DCDQ'07 into the Malayalam language, the DCDQ Malayalam and test its basic psychometric properties.The DCDQ'07 was translated according to the guidelines for the transcultural adaptation of instruments. The DCD questionnaire was initially distributed to parents of 3322 children (5 - 15 years), of which only parents of 602 children completed the DCDQ Malayalam. Out of this total number 55 were retested randomly after 3 weeks in order to assess test retest reliability. The DCDQ Malayalam showed high internal consistency and test-retest reliability. Confirmatory factor analysis showed equivalence to the DCD' Q7. The percentage of probable DCD using DCDQ'0 7 cutoff scores $(\leq 57)$ found out a prevalence of $51.5 \%$. Using more stringent cutoffs $(\leq 38)$ prevalence was found to be $18 \%$.The DCDQ Malayalam provides a promising consent for initial identification of children with DCD who speaks Malayalam language. Based on the more stringent scores the prevalence of children with risk of DCD in the selected district appeared to be around $16 \%$.For more generalization, studies with more samples and comparison with the MABC-2 or equivalent is needed.
\end{abstract}

Index Terms- Developmental coordination disorder, DCDQ, prevalence, psychometric properties.

\section{INTRODUCTION}

DCD is a delay in the development of motor skills or difficulty in coordinating movements which in directly may cause problems in performing sports, daily activities or even academic performance especially in aspects like handwriting. Various studies believe that the child with DCD outgrew as age advances but studies have identified that coordination difficulties in fact worsen with increasing age. Difficulties with DCD may not only hamper their everyday activities but it may even affect their social emotional and behavioural domains of one's life.
However a manifold of studies have proved that functional mobility, participation outcomes and their psycho motor functions could be improved with simple interventions. Guidelines from the European academy for childhood disability (EACD) recommend that, all children identified with DCD should receive intervention(1). This justifies the need of early identification of children with DCD in order to ensure timely intervention thereby preventing secondary complications.

Now many tools are commonly used for the diagnosis of DCD namely the Beery-Buktenica Developmental Test of Visual-Motor Integration (Beery VMI), the Bruininks Oseretsky Test for Motor Proficiency-2 (BOT-2), and the Movement Assessment Battery for Children-2 (MABC-2)(2). Though these tools are popular in research and clinical settings, it is regarded to be costly and is time consuming. This has led to the need for a valid screening test that is economical and less time consuming. Revised DCDQ a parent based testing is one such widely used screening test for the diagnosis of DCD. The instrument has been translated into various languages and adapted for use in several countries including Italy, Taiwan, Canada, and even in India to the Hindi language.

In Kerala, about 19 percentage of the population falls under the age group of 14 years according to the Kerala census 2021. Infant and diseases of children is a major concern in Kerala. DCD is relatively unknown in Kerala. But, it's a commonly linked co morbid condition along with other disorders(3). Attention Deficit Hyperactivity Disorder (ADHD), has a high prevalence rate of $11 \%$ (4). In addition to this, India is the leading country in the world for pre-term births(5) which is also associated with DCD. According to the latest WHO statistics, around three and a half million Indian infants were born prematurely in 2014. Based on this information we could predict a moderate to high prevalence of DCD in Kerala

While the DCD is gaining attention worldwide among the research and clinical areas, in Kerala it is relatively unknown. According to American psychiatric Association, around 10 percentages of School children in United States are affected by $\operatorname{DCD}(6)$. There is a lack of a validated published questionnaire in Malayalam language, which could help in identifying or predictable prevalence of children of Kerala at higher risk of 
DCD. Therefore the aim of this study was to translate the DCDQ in Malayalam language and to test its basic psycho metric properties

\section{Research Elaborations}

The DCDQ'07 was used as the basis to form the Malayalam version DCDQ Malayalam. The DCDQ'07 is a fifteen item parent based questionnaire developed for children between 5 to 15 years. This helps in identifying the fine motor, gross motor and general coordination skills of the children. This instrument is a five-point likert scale for rating motor performance with the total score ranging between 15 and 75 . Total score cut-off ranges for the three age groups (Group 1: 5 - to 7 years, Group 2: 8 - to 9 years and Group 3: 10 - to 15 years) indicates the possibility or probability of child to have $\operatorname{DCD}(7)$.

The DCDQ'07has demonstrated high internal consistency with Cronbach's $\alpha$ value of 0.94 , and item-total correlations ranging from 0.52 - to 0.78.(7) Considering the age-specific cutoffs, it has an overall sensitivity of $85 \%$ and specificity of $71 \%$ (cut-offs for Group $1 \leq 46$, Group $2 \leq 55$, and Group $3 \leq 57$ ). Sensitivity of the DCDQ refers to the percentage of children who are correctly identified as meeting criteria for DCD and specificity is the percentage of children without problems who are correctly identified as such by a screening test(7). Although the DCDQ'07 appears to be a valid screening instrument, additional standardized testing is suggested to confirm initial diagnosis of DCD.

\section{Translation of tool}

Translation of the DCDQ into Malayalam was conducted according to current guidelines for cross cultural adaptation of instruments.

\section{Translation process}

With the permission from the primary test developer, an agreement was signed between the user and the developer. The translation was conducted according to guidelines developed by Beaton et al. This process involved the translation into the standard language and the adjustment of cultural words, idioms and if necessary, complete transformation of some items in order to capture the same concept in the target culture.

\section{Stage 1: Initial translation}

The first stage in the adaptation was the forward translation. Two qualified independent translators with different backgrounds (one with no knowledge about children with coordination disorder and the other one who was aware of the concepts being examined) translated the questionnaire from English to Malayalam (T1and T2 ).Item content, response options and instructions were translated by them. One among the two translators was aware of the concepts being examined in the questionnaire being translated with an intention to provide a more reliable equivalence from a measurement perspective.

\section{Stage 2: Synthesis of the translation}

Working from the original questionnaire as well as the first translator (T1), and the second translator (T2) versions, a synthesis of these translators were conducted producing a common Malayalam version of the DCDQ (T-12). Various issues were addressed regarding the translation of words and its relevance in the culture of Kerala. With regard to the instructions and options, certain difference in the opinion of translation was raised. The options were made into simple form according to the understanding of the people of Kerala. There was certain disparity in the item numbers $6.7,11,12,13,14$ and 15 due to grammatical/word meaning. After analysing the versions of the two translators along with another language expert, an agreement was made in these items based on idioms, grammatic errors and certain words and finally the T-12 version of the questionnaire was developed.

\section{Stage 3: Back translation}

Working from the single Malayalam version of the DCDQ (T-12) and totally blinding to the original version, a back translation to the English language was done by two translators who had their mother tongue as English. The two translators were neither aware nor was informed of the concepts being explored and was without any medical background with an intension to avoid information bias and to elicit unexpected meanings of the items in the translated questionnaire. With regard to question number 14,ambiguity in translation of "Bull in the china shop" was expressed between the two translators as one translator translated it as "bull in China Shop" whereas the other translator translated it as "like an elephant in a sugarcane field." Remaining all other items reflected almost the same item content as the original version.

\section{Step 4: Expert committee}

An expert committee comprising of Pediatric nurses, Paediatricians, Psychologists, Public health experts, Language professional, an occupational therapist and the translators was formed. The material at the disposal of the committee included the original version and each translation (T1 ,T2 T12,BT1,BT2)together with corresponding written reports which explained the rationale of each decision at earlier 
stages.(Annexure-2).The expert committee identified some issues/disparity in item numbers $6,8,9,11,12,14$ and 15 which was rectified after detailed discussion by the committee.

\section{Semantic equivalence}

With regard to semantic equivalence item number $6,8,9,11$ and 15 had certain grammatical difficulties which was clarified and appropriate corrections in the grammar/structure was made.

\section{Idiomatic equivalence}

Item number 14,which consisted of 'BULL IN A CHINA SHOP" was found difficult to translate to the cultural idiom .Therefore another idiom with similar meaning emphasizing on "like the elephant in a sugarcane farm" was found appropriate based on the BT2 and was adopted.

\section{Experiential equivalence}

Item number 12, the questionnaire item contained roller skating and swimming which was not a common activity in the culture and geographical area. The questionnaire item was added by similar item (cycling) which is common in the target/ geographical area.

\section{Conceptual equivalence}

The item number 11, had discrepancy with regard to the item "competition" which was replaced to a word with similar meaning of games. Consensus was reached on the items, instructions and response options. They also agreed with the general recommendation for questionnaire specifying that the final questionnaire is understandable by a 12 year old child. The same questionnaire was administered to a 12 year child who could read it easily and understand the same very well.

\section{Stage 5: Test of the pre final version}

The final stage, the test of the pre final version was conducted among 41 parents of children within the age group of 5to15,administered through Google form (https://forms.gle/8EjtZPbusnUDTDY4A).In view of restriction of Covid 19 pandemic, meeting physically was not possible. All the items were given an extra option to rate that "the question was not understandable". The distribution of responses were examined and was found that all the subject's responded to all the items and had no missing nor single responses. The subjects were personally contacted through phone to review what he/she thought or understood about questionnaire item and the chosen response.

\section{Stage 6: Submission of documentation to the developer or coordinating committee for approval of the adaptation process}

Finally the reports and forms were submitted to the developers of the instrument and the approval was obtained after auditing the process and the steps followed in the translation of instrument

\section{Sample}

The samples for the study were taken from 16 schools of Kozhikode city in which consisted of 3322 parents of children representing 1 st to 7 th standard. Cluster sampling technique was used to identify the schools and convenience sampling was used to identify the children. Initially the consent from the Principals of the schools were taken

\section{Procedure}

Once the translation of the tool was done, the translated DCDQ Malayalam was given for content validity to twelve subject experts. There was $100 \%$ agreement regarding the content of the tool. When the time came for the administration of the questionnaire for the parents due to the covid-19 pandemic, it was unable to give them physically by means of hard copy. The questionnaire was made into a Google form and the link was sent to all the parents of the selected age groups, through the WhatsApp groups created by the School. Those parents who gave the consent in the Google form were allowed to move further in order to fill this questionnaire. A voice message depicting the signs and symptoms that necessitate screening children and the consequences of not identifying the DCD was developed and was distributed along with the Google form. The parents were also informed that the participation was completely voluntary and assured that the completed questionnaire and data would be kept confidential. Telephonic assistance was also provided parents in case of any doubts or clarifications. Among the 3322 parents, 602 parents fill the form and were submitted. 55 parents were selected randomly from the 602 parents and re tested after three weeks from initial screening for test retest reliability.

\section{Statistical analysis}

Descriptive analysis was done to identify the characteristics of children according to gender and age. The total data set was divided into three age groups namely Group 1(5 to 7 years), group 2(8 to 9 years) and group 3(10 to 15 years). Due to the normality appearance of data, parametric test (alpha level $0.05)$ was used for further analysis. Internal consistency of DCDQ Malayalam was assessed using Cronbach's Alpha and corrected item- total correlations was also done (corrected item- 
total correlations were calculated to examine the relationship between each item and the whole questionnaire).The test retest reliability was assessed by means of Pearson's correlation coefficient and intraclass correlation coefficient(ICC) for all 15 items. Confirmatory factor analysis was done to test the structure of the DCDQ Malayalam. The cut off scores as described in the DCDQ were used to identify those children to have indication of, or suspect for DCD, as same as that of the DCDQ manual. In line with the DCDQ '07, 15 questions in the DCDQ Malayalam were divided into three subsets namely control during movement, general coordination and handwriting.

\section{Results or Finding}

\section{Sample characteristics}

\section{Table1: Sample characteristics}

\begin{tabular}{|c|c|c|c|}
\hline S1 & Sample characteristics & Frequency & Percentage $(\%)$ \\
\hline & $\begin{array}{l}\text { Age of the child } \\
\begin{aligned} \text { - } & 5-7 \text { years } \\
\text { - } & 8 \text { to } 9 \text { years } \\
\text { - } & 10 \text { to } 15 \text { years }\end{aligned}\end{array}$ & $\begin{array}{l}175 \\
184 \\
243\end{array}$ & $\begin{array}{l}29 \\
31 \\
40\end{array}$ \\
\hline & $\begin{aligned} \text { Gender } & \\
& \\
\text { - } & \text { Male } \\
\text { - } & \text { Female }\end{aligned}$ & $\begin{array}{l}321 \\
281\end{array}$ & $\begin{array}{l}53 \\
47\end{array}$ \\
\hline
\end{tabular}

The tool was initially administered to 3322 parents of children within the age group of 5 to 15 years. Among them, only 602 parents completed the questionnaire. No obvious patterns of missing items were identified.

\section{Reliability}

\section{Internal consistency}

Inter item correlation; item total correlation and Cronbach's alpha were used to determine the consistency of the DCDQ Malayalam. The item total correlation coefficient ranged from (0.48-1.00). The total Cronbachs alpha was 0.80 which was above the acceptable level of 0.70 indicating a high internal consistency. When individual items were deleted alternately all item deleted Cronbach's alpha values were lower than the value of 0.80 . Subset 1 and subset 2 showed moderate internal consistency $(\alpha=0.56 \& 0.60)$ while subset 3 showed $(\alpha=0.70)$ high internal consistency. All items were moderately correlated (0.48-1.00). Since Cronbach's alpha did not increase on deletion of any of the 15 items all items were considered to be positively correlated, no item was omitted.

\section{Test retest reliability}

From the total sample, 60 participants were retested and were considered for analysis using Pearson's correlation. Five out of 60 questionnaires were excluded as they had either missing entries or incomplete data. Pearson's coefficient was 0.73, which indicated moderate reliability. Pearson's coefficient was 0.74 for subset1,0.63 for subset 2 and 0.63 for subset 3 .

\section{Construct validity:}

Construct validity was determined by means of confirmatory factor analysis. The 3-factor solution was used with the DCDQ'07 namely 'control during movement', fine motor/Handwriting and general coordination', as the initial model for conducting confirmatory factor analysis of the DCDQMalayalam. The 15 items of the DCDQ were subjected to principal component analysis (PCA) using SPSS. Prior to performing PCA, the suitability of the data for factor analysis was assured by means of Kaiser Meyer Olkin measure and Bartletts test of sphericity of sampling adequacy. The appropriateness of factor analysis was supported by Barletts test of sphericity, an indicator of the strength of relationship among variables. It was found that the results were $\operatorname{significant}\left(\chi^{2}\right.$ =262.106). The KMO measures of sampling adequacy yielded a value of 0.64,indicating that the sample size was appropriate to assess the factor structure. Finally the communalities of items

ranged from 0.53 to 0.74 . Table 2 shows the KMO, communalities and Barletts test.

TABLE 2:The KMO, communalities and Barletts test 


\begin{tabular}{|l|l|}
\hline TEST & RESULT \\
\hline $\begin{array}{l}\text { Barlet's test of sphericity } \\
\text { approximation } \chi^{2}\end{array}$ & 262.106 \\
\hline $\begin{array}{l}\text { Kaiser Meyer Olkin (KMO) } \\
\text { measure of sampling } \\
\text { adequacy }\end{array}$ & 0.64 \\
\hline df & 105 \\
\hline Significance & 0.00 \\
\hline Communalities (Range) & 0.53 to 0.74 \\
\hline
\end{tabular}

\begin{tabular}{|l|l|l|l|}
\hline 9 & .709 & 4.724 & 81.064 \\
\hline 10 & .659 & 4.394 & 85.458 \\
\hline 11 & .594 & 3.963 & 89.421 \\
\hline 12 & .510 & 3.401 & 92.822 \\
\hline 13 & .423 & 2.817 & 95.639 \\
\hline 14 & .381 & 2.542 & 98.181 \\
\hline 15 & .273 & 1.819 & 100.000 \\
\hline
\end{tabular}

Given these overall indicators ,EFA was then conducted with 15 items using Principal component analysis extraction and Varimax rotation. The minimum factor loading cut off point for this study was 40 . The six factors explaining 64.93 percent of the variance was identified. The Eigen value showed that the first factor explained 18.949 percent of the variance, the second factor 12.170 percent of the variance and the third factor 10.203 of the variance.

\begin{tabular}{|c|c|c|c|}
\hline \multicolumn{2}{|c|}{ Component Total } & $\%$ of Variance & Cumulative \% \\
\hline 1 & 2.842 & 18.949 & 18.949 \\
\hline 2 & 1.826 & 12.170 & 31.120 \\
\hline
\end{tabular}

Table 3: Intra class Correlation Coefficient of the two tools

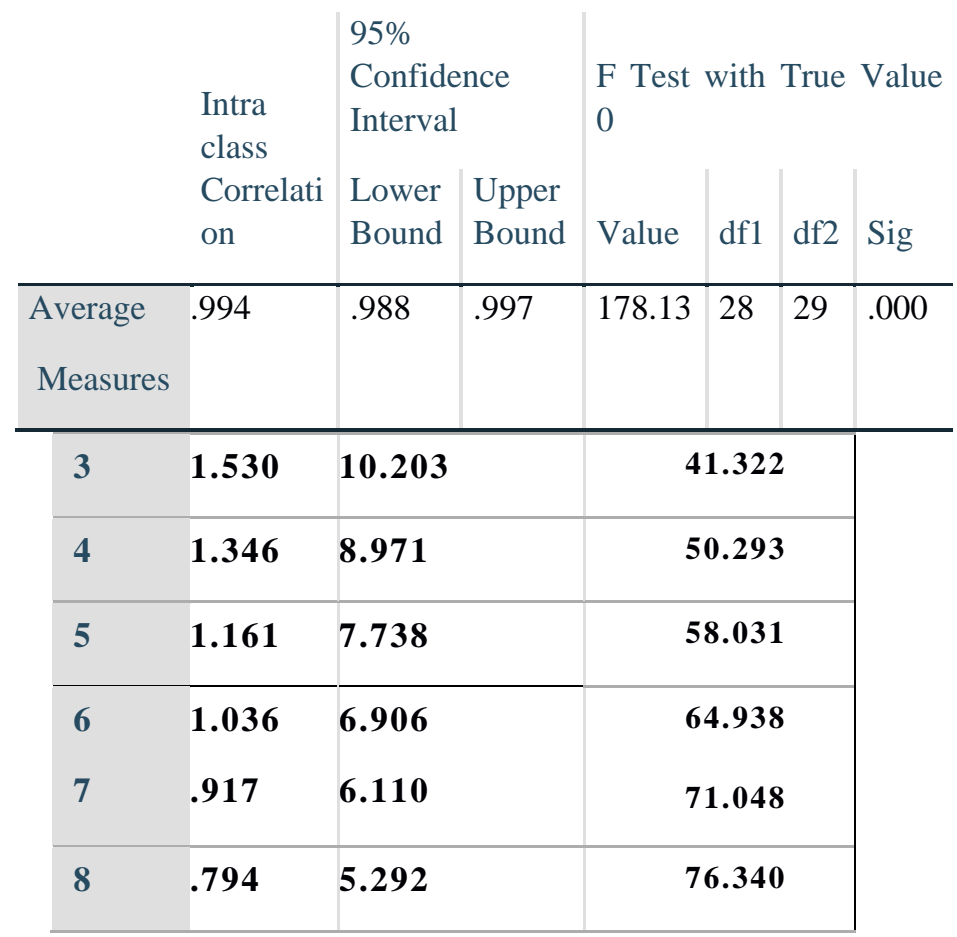

\section{Convergent validity}

In order to check the convergent validity, 30 parents who were experts in both the languages (Malayalam and English) were selected and was asked to fill both the tools.

TABLE 2:Mean and Standard deviation of two version tools

\begin{tabular}{ll|l|l|l|} 
& $\mathrm{n}$ & Minimum & Maximum & Mean \\
\hline Malayalam & 29 & 23 & 64 & 48.24 \\
\hline English & 29 & 23 & 66 & 48.31 \\
\hline
\end{tabular}

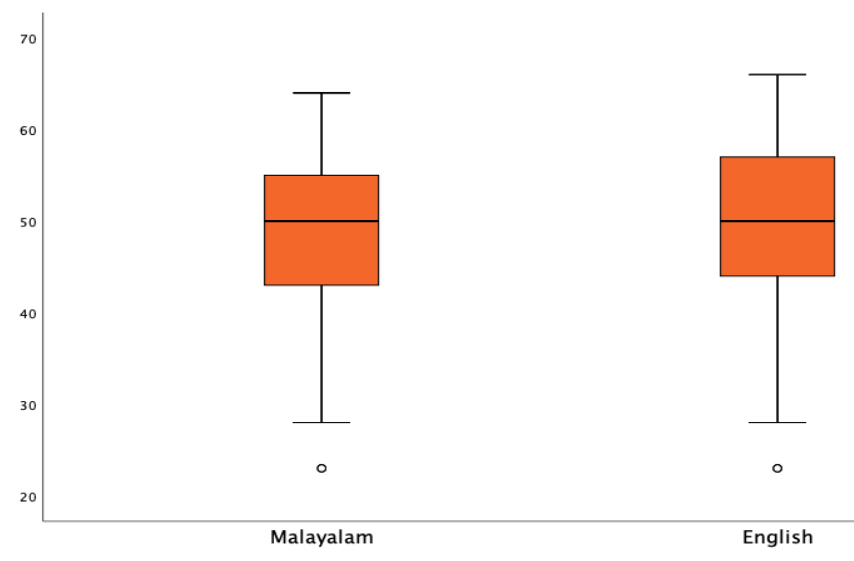

\section{Estimation of probable DCD.}

Of the total 602 participants who have responded, 310 were identified to have indication of or suspect for DCD. Percentage of probable DCD was found to be $51.5 \%$ across the three age groups. This estimated an average prevalence rate of $9 \%$. Detailed information on total number of indication 
ISSN 2250-3153

or suspect for DCD across the three age groups is described in table 4

Table 4: Total number of children of indication or suspect for DCD

\begin{tabular}{|l|l|l|l|}
\hline Age group & $\begin{array}{l}\text { Indication of or } \\
\text { suspect } \\
\text { for DCD }\end{array}$ & $\mathrm{f}$ & $\%$ \\
\hline $\begin{array}{l}5 \text { years to } 7 \text { years } \\
11 \text { months }\end{array}$ & 15 to 46 & 45 & 14.52 \\
\hline $\begin{array}{l}8 \text { years 0 months } \\
\text { to years 11 } \\
\text { months }\end{array}$ & 15 to 55 & 106 & 34.19 \\
\hline $\begin{array}{l}10 \text { years 0 months } \\
\text { to } 15 \text { years }\end{array}$ & 15 to 57 & 159 & 51.29 \\
\hline
\end{tabular}

\section{Discussion}

The purpose of this study were to translate the DCDQ into the Malayalam language, test its basic psychometric properties and provide a preliminary report of probable prevalence of children with high risk for DCD in Kerala. This is considered to be the first adaption of the DCDQ'07 to the language of Kerala-Malayalam. As reported by the parents and the experts during the field testing, the questionnaire was relatively easy to understand and culturally adaptable. In addition to being appropriate of use in the general culture of Kerala, the instrument illustrated acceptable equivalence to the DCDQ'07.As per the mentality of parents to refrain from providing information related to their child's developmental status, validity is a major concern. Studies have figured out that parent completed developmental questionnaires provide relatively accurate information about motor development and can be considered to be more valid and reliable. This study has demonstrated that the selected psychometric properties of the DCDQ Malayalam are comparable to other cross cultural studies of translated versions of the DCDQ'07.The analysis showed a high internal consistency $(\alpha=0.80)$ which suggests that all items in the DCDQ Malayalam are homogenous for measuring motor coordination. Correlation analysis showed a moderate positive correlation between individual item values and total questionnaire score. (0.48-1.00).

Results of the 3 factor Confirmatory factor analysis model were comparable to that of the DCDQ'07.The model which best fit included the following three factors namely control during movement, Fine/Motor Handwriting and general coordination.

The major finding of this study was the identification of children with probable DCD and more probable DCD.Based on the cutoff scores suggested in the DCDQ'07 instructions,a high rate of children at risk for DCD was found, the average cross ages was 51.5\%.After using the more stringent cut-off scores of $\leq 38$, the prevalence rate reduced to $18 \%$. A similar discrepancy was illustrated by Caravale etal (8),when using the $15^{\text {th }}$ percentile instead of the $5^{\text {th }}$ percentile of the MABC-2.The cross cultural adaptation of the DCDQ'07 into Chinese language also used different cut off scores. So it could be reasonable to consider these cut-off scores in initial identification of children at risk of DCD. However these results have to be validated further using objective tests namely Movement Assessment Battery for children-2 or Motor Screening assessment tool (MSA).

The study has certain limitations. First of all, there was no use of objective tests to confirm the children to suspect for DCD. Moreover, Kozhikode being just a part of the Kerala state, screening in different parts of the state is needed to generalize this result.

\section{Conclusion}

The Malayalam version of DCDQ could be used as an initial screening instrument to rule out children with DCD. The DCDQ Malayalam demonstrated satisfactory internal consistency and reliability. Future studies could include verification of DCDQ Malayalam with objective tests (MABC-2, MSA or BOT) in various areas of Kerala and with a larger population. Based on the findings of the study the use of more stringent cut off scores will help to determine the most accurate prevalence of DCD in a state like Kerala. 


\section{ACKNOWLEDGMENT}

MY DEEP SENSE OF GRATITUDE IS EXTENDED TO THE CHILDREN, PARENTS AND THE PRINCIPALS OF ALL SCHOOLS FOR THEIR SUPPORT AND HELP RENDERED DURING THE STUDY.

\section{REFERENCES}

[1] R. Blank et al., "International clinical practice recommendations on the definition, diagnosis, assessment, intervention, and psychosocial aspects of developmental coordination disorder," Dev. Med. Child Neurol., vol. 61, no. 3, pp. 242-285, Mar. 2019, doi: $10.1111 /$ dmcn.14132.

[2] P. M. M. Okuda, M. Pangelinan, C. Chiorri, S. A. Capellini, and H. Cogo-Moreira, "A new motor screening assessment for children at risk for motor disorders: Construct validity," Arq. Neuropsiquiatr., vol. 76, no. 2, pp. 104-112, Feb. 2018, doi: $10.1590 / 0004-282 \times 20170183$.

[3] "Kerala Population 2021." https://www.indiacensus.net/states/kerala (accessed Oct. 18, 2021).

[4] Venkata and A. S. Panicker, "Prevalence of attention deficit hyperactivity disorder in primary school children," Indian J. Psychiatry, vol. 55, no. 4, p. 338, Oct. 2013, doi: 10.4103/0019-5545.120544.

[5] H. Blencowe et al., "Preterm birth-associated neurodevelopmental impairment estimates at regional and global levels for 2010," Pediatr. Res. 2013 741, vol. 74, no. 1, pp. 17-34, Dec. 2013, doi: 10.1038/pr.2013.204.

[6] American Psychiatric Association, American Psychiatric Association, 2013. Diagnostic and statistical manual of mental disorders (5th ed.). 2013.

[7] B. N. Wilson, S. G. Crawford, D. Green, G. Roberts, A. Aylott, and B. J. Kaplan, "Psychometric Properties of the Revised Developmental Coordination Disorder Questionnaire," Phys. Occup. Ther. Pediatr., vol. 29, no. 2, pp. 182-202, Jan. 2009, doi:

$10.1080 / 01942630902784761$.

[8] B. Caravale, S. Baldi, L. Capone, F. Presaghi, U. Balottin, and M. Zoppello, "Psychometric properties of the Italian version of the Developmental Coordination Disorder Questionnaire (DCDQItalian)," Res. Dev. Disabil., vol. 36, pp. 543-550, Jan. 2015, doi: 10.1016/J.RIDD.2014.10.035.
First Author - Prof Mrs Elizabeth Varkey, Department of Child Health Nursing, Baby Memorial College of Nursing, Kozhikode, Kerala. email id: elzaboby@gmail.com

Second Author -Prof (Dr) Roy K George,Academic director, Baby memorial Hospital,Principal,Baby memorial College Of Nursing, Kozhikode, Kerala.

email id:roykgeorge1966@gmail.com 